\title{
DIMENSIONAMIENTO DE UN SISTEMA DE ENERGÍA TERMOSOLAR MEDIANTE EL USO DE UN MODELO
}

\section{Arturo Daniel Alarcón Rodríguez}

\section{RESUMEN}

En el presente artículo se expone el método de dimensionamiento de sistemas termosolares mediante el uso de un modelo matemático. Este método es comúnmente usado debido que es simple, flexible pero a la vez muy potente. La simulación del sistema termosolar se realiza en base a un modelo matemático que describe los fenómenos térmicos que ocurren mediante un conjunto de ecuaciones diferenciales. Los parámetros que determinan el modelo son coeficientes de intercambio de calor entre los elementos del sistema, parámetros que representan las características de los componentes del sistema termosolar y parámetros que representan las condiciones en las que trabajará el sistema. Estos parámetros se determinan en base a recomendaciones de bibliografía, observaciones, mediciones de campo y correlaciones adecuadas. El uso de un modelo para el dimensionamiento de un sistema termosolar resulta una herramienta muy útil, ya que se adapta a distintas configuraciones de sistemas termosolares. Permite asimismo, tener una idea bastante aproximada del comportamiento del sistema termosolar en distintas condiciones de uso, la que sólo podría obtenerse a través de experimentos físicos complicados y por ende costosos.

Palabras Clave: Sistema Termosolar, Dimensionamiento, Modelo Matematico. 